\title{
HEADHOOD OF SUFFIXES AND FINAL COMBINING FORMS IN ENGLISH WORD FORMATION*
}

\author{
TVRTKO PRĆIĆ \\ Department of English, Faculty of Philosophy \\ University of Novi Sad \\ Dr Zorana Đinđića 2 \\ 21000 Novi Sad \\ Serbia \\ tprcic@EUnet.yu
}

\begin{abstract}
This paper offers a multi-layer description of the morphological, phonological, syntactic and semantic aspects of the behaviour of suffixes and final combining forms which function as heads in morphologically complex and compound words in English, both established and new ones. In the discussion, the following topics will also be dealt with in some detail: (a) a distinction between classical and modern final combining forms, (b) a distinction between classical, modern and dummy linking vowels, (c) dual headedness of composite FCFs, in which a micro-head and a macro-head can be observed to interact, (d) a contentbased approach to construing the headhood of suffixes and final combining forms, and (e) the notion of conceptual endocentricity.
\end{abstract}

Keywords: suffix, final combining form, micro-head, macro-head, conceptual endocentricity

\section{Introduction: setting the scene}

The aims of this paper are twofold: firstly, to describe the behaviour of suffixes and final combining forms (henceforward, FCFs) which appear as heads in morphologically complex and compound words; and secondly, to point out some differences between their behaviours. In order to bring out the latest developments as well, the account draws on the observation of not only established and well-known words but also of new vocabu-

* For very useful remarks and suggestions on an earlier version of this paper I wish to thank an anonymous reviewer; thanks are also due to the participants in the discussion at the 12th International Morphology Meeting (Budapest, May 2006). 
lary, mostly electronically available (see particularly www.onelook.com and its very useful wildcard search facility). (For various treatments of suffixes and FCFs, see Adams 1973; 2001; Back 1991; Bauer 1983; 1998; Dalton-Puffer-Plag 2000; Huddleston-Pullum 2002; Kastovsky 1986; Lehrer 1998; Ljung 2003; Marchand 1969; McArthur 1992; Plag 2003; Prćić 1999; Quirk et al. 1985; Stein 1977; Tournier 1993; Warren 1990.)

Building around the sign-oriented conception of word formation (Marchand 1969), both suffixes and FCFs are seen as synchronically separable, bound right-hand input elements, with an identifiable form, content and function. The term 'separable' (after Guierre, in Tournier $1993,51)$ emphasizes the fact that the bound element has a status of its own, because "a particular phonological form is systematically associated with at least one particular content and one particular function" (Prćić 1999, 266), "which enables it to be separated, or detached, from the other element(s) in a word without that bound element's form, content and function being blurred, obscured or otherwise rendered unrecognizable" (Prćić 2005, 315). Therefore, -er and -ish, for example, are suffixes in teach.-er and green.-ish but not in barter and finish, whereas -logy and -holic are FCFs in morpho-.-logy and fooda-.-holic but not in apology and alcoholic (the hyphen indicates the boundness of an element and the dot within a word the boundary between the two immediate input elements).

Suffixes, in their prototypical manifestation, will be defined here as bound right-hand input elements which occur with free bases only and have functional/lexical meaning of low semantic density, i.e., high generality. Such are, for example: wash.-able, magic.-al, bankrupt.-cy, employ.-ee, teach.-er, child.-hood, solid.-ify, yellow.-ish, violin.-ist, attract.-ive, enjoy.-ment, kind.-ness, poison.-ous, friend.-ship, milk.-y.

On the other hand, FCFs (which were first introduced, together with initial combining forms, or ICFs, by Bauer 1983) in their prototypical manifestation, will be defined as bound right-hand input elements which occur with ICFs, prefixes and free bases, and have full lexical meaning of high semantic density, i.e., high specificity. According to their origin (etymology and derivation combined), two broad categories of FCFs can be differentiated: the first involves classical FCFs (henceforward, CFCFs), which are formal, semantic and etymological varieties of classical (Greek and Latin) words or elements in their appropriate senses, like morpho-.-logy for science, photo-.-graphy for writing, hydro-.-phobia for fear, demo-.-cracy for government, biblio-.-phile for lover (of), regi-.-cide for killing and killer. The second category com- 
prises modern FCFs (henceforward, MFCFs), which are formal and semantic varieties of modern English words or elements (sometimes borrowed from other modern languages) in their appropriate senses. There are two types of MFCFs:

(1) Extractions produced by front-clipping, often as a result of repeated superimpositional blending, which gradually develops into a pattern. For example, the MFCF -holic has been extracted from the source word alcoholic by the superimposition of elements like work $(+a)$, shop $(+a)$, choco(late) and others onto the initial part of the source word (alco), thus isolating its latter part (holic), with the meaning 'person addicted to something', which is wider than that of the source word (alcoholic) (for an innovative approach to blending, see Kemmer 2003). The following are currently the most frequent extractions - some of them fully established and some only partly so (they are listed within their source words, where the superimposee elements are separated by a slash): ham/ -burger, caval/-cade, broad/-cast, Water/-gate, tele/-gram, alco/-holic, auto/-mobile, astro/-naut, eco/-nomics, encyclo/-pedia, lite/-rati, land/ -scape, new/-speak, mara/-thon, soft/-ware. As will be noted, some of the source words from which these MFCFs were extracted (telegram, automobile, astronaut, economics, encyclopedia, marathon) are of classical origin, but the creations themselves and their meanings are modern - in fact, they have originated quite recently. Here are now examples of each of the extractions above: cheese-burger, auto-cade, web.-cast, Monica.-gate, kisso-.-gram, golfa-.-holic, snow.-mobile, info.-naut, Thatcher.-nomics, webo-.-pedia, glitte[r].-rati, river.-scape, net.-speak, swima-.-thon, free. -ware (the letters within square brackets indicate that those letters are dropped).

It might be interesting to mention that at present the citation forms of three extractions are found in several random variations: -holic also occurs as -aholic and -oholic, -rati as -erati, and -thon as -athon, with these apparent allomorphs largely going unexplained, both in the literature and in dictionaries. There is indeed a much greater number of instantiations of the longer forms of these MFCFs in words (golf-.--?aholic), where the initial vowels provide a smooth and euphonic transition between the two input elements. However, with the existence of words like pepsi.-holic, coffee.-holic, sugar.-holic, techno-.-rati, digi-.-rati, cultu[re]-.-rati, tele-. -thon, radio.-thon, boobie.-thon, whose left-hand input elements already contain final transition-providing vowels, it seems reasonable and more justified, until a firm(er) system of division of labour between the forms 
of these MFCFs emerges, to opt for the shorter varieties as their citation forms and to assign to the initial vowels, when they appear in the structure, the role of a linking vowel-classical, modern or dummy, which properly belong to the left-hand input element (and which will be dealt with later). (For a discussion of -holic and -thon, see Lehrer 1998.)

(2) Various other bound forms: scandal.-monger, including borrowings from other modern languages: gab.-fest, horror.-meister, dulls-.-ville, which are free-standing words in their source languages - the first two coming from German and the last from French.

As bound input elements, both suffixes and FCFs are non-viable by themselves and hence they require companion left-hand input elements: to return now to the examples from the very beginning, the suffix -er requires the base teach and the FCF -logy requires the ICF morpho-. Together with their companion left-hand input elements, both suffixes and FCFs produce output formations of a binary structure, in which the right-hand element acts as the head (theme) of the formation and the left-hand element as the modifier (rheme) of the head: in teacher, -er is the head and teach its modifier, and in morphology, -logy is the head and morpho- its modifier.

The head influences the makeup of the output formation in four respects - morphological, phonological, syntactic and semantic. In what follows, a separate section will be devoted to each one of them.

\section{Morphological aspects of suffix/FCF headhood}

Morphologically, the suffix/FCF head determines the final shape of the output word, to which new lexical and/or grammatical suffixes can be added.

Suffixes regularly end the words in which they appear and they are, similar to prefixes, recursive in nature: modern.-iz[e].-ation, transform. -ation.-al.-ly. In contrast, FCFs also end the words in which they appear but they are, unlike ICFs, not recursive in nature: lexico-.-logy, shopa-.-holic. Some FCF, both classical and modern, like phobia, ology (with the linking vowel retained; for discussions of its status, see Prćić 2005; Bauer 1998), burger, pedia, meister, have become unbound lexical elements, because of their high semantic density, which has enabled their independent use, their subsequent base-like behaviour and, ultimately, their full free-standing base status. 
From the point of view of right-hand-left-hand input elements cooccurrence restrictions, suffixes occur with free input elements only, i.e., bases: lion.-ess, nice.-ly, modern.-ize, while FCFs occur with bound input elements, i.e., ICFs and prefixes, and with free input elements, i.e., bases, as follows:

- $\mathrm{CICF}^{1}+\mathrm{CFCF}$ : morpho-.-logy, anthrop[o]-.-onym;

- CICF + MFCF: biblio-.-holic, anglo-.-holism (this word is the author's own coinage, in full 'linguistic angloholism', denoting the final, extreme phase of linguistic anglomania, so widespread in the world today);

- MICF + CFCF: futuro-.-logy, speedo-.-meter;

- MICF + MFCF: talka-.-thon, milka-.-holic;

- prefix + CFCF: cyber-.-phobia, mono-.-chromatic;

- prefix + MFCF: multi-.-cast, tele-.-thon;

- base + CFCF: Beatle.-mania, web.-centric;

- base + MFCF: free.-ware, zipper.-gate.

As can be seen, some FCFs, especially CFCFs, can occur with several types of left-hand input elements (CICFs, MICFs, prefixes and bases): -phile, -phobe, -phobia, -mania, -logy, -cracy, -meter, -centric (CFCFs), -holic, -pedia (MFCFs). This "interbreeding" has been made possible by the fact that such CFCFs have moved from the scientific register, in which they were predominantly used, to the general, non-specialized vocabulary, and also because of a dwindling interest in, and consequently the teaching of, the classical languages in many of present-day educational systems.

Also illustrated by some of the examples above is a novelty regarding the linking vowel. Namely, alongside, and occasionally instead of, the prototypical linking vowel $-o-$, there is an increasing use of the homophonous -a-, mainly in new words, like milka-.-holic, shopa-.-holic, worda-.-holic, strippa-.-gram vs. strippo-.-gram, weba-.-holic vs. webo-.-holic, choco-. -holic vs. choca-.-holic. Sometimes both linking vowels are ambihyphenated in words: shop-a-holic, choc-a-holic and spend-o-holic, film-o-holic, or even capitalized within them: PlaceOPedia. Nevertheless, neither of these spelling practices appears to be standard English (yet) and it re-

${ }^{1}$ Similar to FCFs, ICFs are also divided into classical ICFs (CICFs), formal, semantic and etymological varieties of classical (Greek and Latin) words or elements in their appropriate senses, and into modern ICFs (MICFs), formal and semantic varieties of modern English words or elements in their appropriate senses. (For a detailed discussion of this and of certain CICFs proposed for reassignment to the category of prefixes, see Prćić 2005.) 
mains to be ascertained by careful observation to what extent and in what registers these usages will catch on, as well as how the distribution between the two linking vowels will evolve. In the meantime, the emergence of a new (allographic variety of) linking vowel demands a name for the element - if - $o$ - is a classical linking vowel (in terms of its origin), then the new - $a$ - obviously warrants the designation modern linking vowel.

Not belonging to this category are vowels occurring finally in lefthand input elements of words like sugar.-holic, digi-.-rati, tele-.-thon, stripper.-gram, Thatcher.-nomics. All these vowels provide a smooth and euphonic transition between the left-hand and the right-hand input elements of a word, but, besides mere phonological form, they have no morphemic status. As a result, unlike the classical linking vowel - 0 - (and $-i-$, as in agri-.culture) and the modern linking vowel $-a-$, which have both morphological and phonological realizations, all other vowels can at best be qualified as dummy linking vowels, because they function as a sort of linking vowel - phonologically only but not morphologically (cf. bureaucracy, which contains a dummy linking vowel, coinciding phonologically with the classical $-o-$ ).

From the structural point of view, the addition of new lexical suffixes to words ending in a suffix or an FCF has different implications. Among suffixes there are no composite suffixes: -ability, -fulness, -ically, etc. are only suffix combinations with a compositional meaning (i.e., $-f u l+-n e s s)$, whereas the suffixes -manship, -ician and -ical represent fusions with unique, non-compositional meanings. The suffix -manship, isolated from words like workman.-ship and craftsman.-ship, unlike the suffix -ship, means 'skill in an activity, especially of a competitive nature' (cf. Random House Webster's Unabridged Dictionary (1999); MerriamWebster's Collegiate Dictionary (2003)) and is added to bases which do not end in -man: brink(s).-manship, grants.-manship, quotes.-manship, oneup.-manship, life-manship; the suffix -ician, synonymous with the nominal senses of -an and -ian, is added to bases which do not end in -ic: diet.-ician, beaut[y]-.-ician (there are no bases *dietic or *beautic); the suffix -ical, synonymous with -ic (and the adjectival sense of -al), is added to bases which do not end in -ic: nonsens[e].-ical, farc[e].-ical, -quizz.-ical, -index.-ical (there are no bases ${ }^{*}$ nonsensic, ${ }^{*}$ farcic, ${ }^{*}$ quizzic or *indexic).

In contrast, FCFs, and especially CFCFs, are subject to suffixal expansion, typically into adjectives, with the suffixes $-i c$, -al and -ous, but also into adverbs, nouns and verbs, as in (tele-.)-scopical.-ly, (photo-.) 
-graph[y].-er, (mytho-.)-log[y].-ize, respectively (in some cases, like -cephalic and -cephalous, no synchronically related input nominal FCF is available). In this way, a composite FCF is produced, which, it has to be emphasized, is not unitary in nature but compositional, with a recursive capacity of the process: (morpho-.)-logy vs. -logic, -logical, -logically, -logist, (carni-.)-vore vs. -vorous, -vorously, -vorousness. Even though it is the word as a whole to which a suffix is formally and semantically added, the addition takes place on the FCF, the head of the word, when the added suffix turns into the head of the composite FCF and the bare FCF becomes its modifier: $-\log [y] .-i c$. As a result, such words can be seen to display dual headedness - one at the level of the word as a whole, the other at the level of the composite (expanded) FCF. The head at the composite FCF level (-ic) will be termed the micro-head and it is nested within the head at the word level, the macro-head (-logic). In cases of multiple suffixal expansions, like (morpho-.)-logically, this head-withinhead nesting manifests itself cyclically and hierarchically with each iteration. The dual headedness view just outlined is in keeping with the binary head-modifier principle and is properly to be construed as a matter of perspective: at the more general level, the word as a whole is highlighted; at the more specific level, the composite FCF is spotlighted.

\section{Phonological aspects of suffix/FCF headhood}

Phonologically, the suffix/FCF head determines the final stress placement in the output word, depending on whether the head is stress-neutral or stress-imposing.

When suffixes are stress-neutral, they leave the placement of primary stress on the input base, as in 'wash.-able, 'teach.-er, 'child.-hood, and when stress-imposing, they attract primary stress either onto themselves: employ.-'ee, or onto the penult: $a^{\prime} t o m .-i c$, or onto the antepenult: so'lid.-ify.

In contrast, CFCFs are typically stress-imposing, forcing primary stress to fall on the third syllable from the end of the word, i.e., on the antepenult (Ljung 2003, 146), as in mor'pho-.-logy, pho'to-.-graphy, car'ni-.-vorous. In composite CFCFs, stress placement is governed by the suffixes employed - specifically, by the last (rightmost) suffix, which operates according to its stress-neutral or stress-imposing nature, thus overriding the above third-syllable-from-the-end principle of bare CFCFs: morpho-.-'logical, photo-.-'graphic (both are stress-imposing), car'ni-. 
-vorousness (stress-neutral). MFCFs display various phonological patterns: extractions typically inherit the stress placement of their source words and are mostly stress-neutral, with the exception of -holic, -nomics, -pedia and -rati, which attract primary stress onto themselves: shopa-. -'holic, burger.-'nomics, Wiki.-'pedia, cultu/re]-.-'rati. The MFCFs -monger, -fest, -meister, -ville are all stress-neutral, as in 'rumour.-monger, 'talk.-fest, 'puzzle.-meister, 'nowheres-.-ville. (For details of the phonological behaviour of individual suffixes and FCFs, see Wells 2000; Jones 2006.)

\section{Syntactic aspects of suffix/FCF headhood}

Syntactically, the suffix/FCF head determines the final syntactic category, i.e., part of speech, of the output word as well as its semantics- and pragmatics-related subcategorization.

Suffixes perform either a category-changing function, whereby the input base and the output word belong to different syntactic categories, like: broad $_{\mathrm{adj}}>$ broad.-en $_{\mathrm{v}}$, sing $_{\mathrm{v}}>$ sing.-er $_{\mathrm{n}}$, music $_{\mathrm{n}}>$ music.-al $_{\mathrm{adj}}$, or a category-preserving function, whereby the input base and the output word belong to the same syntactic category, like: leader $_{\mathrm{n}}>$ leader.-ship $_{\mathrm{n}}$,

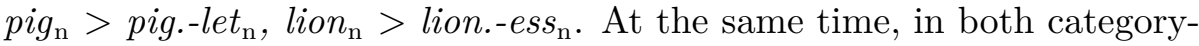
changing and category-preserving cases, there is implied information of the subcategorization of any such word, which takes place within the given syntactic category and which is conditioned mostly semantically and pragmatically. For example, in sing.-er $r_{n}$, the implication contributed by the suffix is 'concrete (noun)', and in $\operatorname{lion}_{\mathrm{n}}$ vs. lion.-ess $\mathrm{n}$, it is the distinction between 'masculine (noun)' and 'feminine (noun)'. (For a different account of the syntactic effects of suffixes, see Huddleston-Pullum $2002,1667-8$.)

In contrast, FCFs perform neither category-changing nor categorypreserving functions, because this feature does not pertain to FCFs. Instead, they function as (a kind of) nouns, adjectives and verbs, as in: morpho-.-logy $y_{\mathrm{n}}$, helio-.-centric $\mathrm{adj}_{\mathrm{j}}$, colour.-cast $\mathrm{v}_{\mathrm{v}}$. This property of FCFs is comparable to that of free bases in ordinary compounds: boat.house $e_{\mathrm{n}}$, sugar.free $_{\mathrm{adj}}$, chain.smoke . $_{\mathrm{v}}$. 


\section{Semantic aspects of suffix/FCF headhood}

Semantically, the suffix/FCF head, firstly, determines the final compositional meaning of the output word and, secondly, it establishes the relationship of hyponymy between itself and the word.

Suffixes have functional/lexical meaning of nominal, adjectival, verbal and adverbial nature, in accordance with their syntactic function, as in: happy.-ness $\mathrm{n}_{\mathrm{n}}$, care.-ful $\mathrm{adj}_{\mathrm{j}}$, modern.-ize $\mathrm{v}$, warm.-ly $\mathrm{ydv}_{\mathrm{adv}}$. The meaning of suffixes is typically of low semantic density and it constitutes the semantic pivot of the word, but it is underspecified on its own (-ness) and thus in need of being specified by the meaning of the modifier (happy).

In contrast, FCFs have full lexical meaning of nominal, adjectival and verbal nature, as in: morpho-.-logy $y_{\mathrm{n}}$, helio-.-centric $c_{\mathrm{adj}}$, colour.-cast $t_{\mathrm{v}}$. The meaning of FCFs is of high semantic density and it constitutes the semantic pivot of the word, but it is incomplete on its own (-logy) and thus in need of being completed by the meaning of the modifier (morpho-). ${ }^{2}$ This property of FCFs is comparable to that of free bases in ordinary compounds.

As for the relationship of hyponymy between the word and its head, the head acts as a conceptual superordinate of the hyponymous word. In the word king.-dom, for example, the suffix -dom is its head, which expresses 'territory'. This basic principle has given rise to an extension of the standard hyponymy test, $\mathrm{X}$ is a kind/type of $\mathrm{Y}$ (Cruse 1986), to be used in describing the headhood of suffixes and FCFs in morphologically complex and compound words. The modified test has the following form:

$$
\mathrm{X} \text { is a kind of (Y, which expresses) Z, }
$$

where X stands for any word incorporating a suffix or FCF (king.-dom), $\mathrm{Y}$ for the suffix or FCF (-dom), and Z for its meaning ('territory'). According to this two-tier analysis, the formal head of the word - non-viable by itself, due to its bound nature - is construed through its content, the general concept which it expresses: king.-dom is a kind of -dom (the low-priority first tier, capturing the bound form), which expresses 'territory' (the high-priority second tier, capturing the bound form's con-

2 The use of the terms 'underspecified' and 'specified', on the one hand, and 'incomplete' and 'completed', on the other, reflects a substantial difference: the first pair presupposes low semantic density (i.e., high generality of meaning), whereas the second pair presupposes high semantic density (i.e., high specificity of meaning). 
tent) - or, to focus on the second tier only, with the parentheses removed: king.-dom is a kind of territory. The method of analysis put forward here will be called a content-based approach to suffix/FCF headhood. It has been conceived of primarily as a counterpart to form-based approaches, which are neatly applicable to most endocentric compounds (like school.teacher is a kind of teacher) and most endocentric prefixations (like micro-.computer is a kind of computer), but not nearly as neatly to words containing suffixes and FCFs (for insightful descriptions, see Williams 1981; Bauer 1990; Kastovsky 1986).

This approach, as can be inferred from the example above, builds around a metalinguistic, noun-centred paraphrase of the concept expressed. Here are some additional examples: for nominal suffixes: -lion. -ess is a kind of (-ess, which expresses) 'female'; for verbal suffixes: modern.-ize is a kind of (-ize, which expresses) 'causative activity'; for adjectival suffixes: care.-ful is a kind of ( $-f u l$, which expresses) 'property'; for adverbial suffixes: nice.-ly is a kind of (-ly, which expresses) 'manner of doing things'. The paraphrases are similar for CFCFs: morpho-.-logy is a kind of (-logy, which expresses) 'science', for extractions within MFCFs: kisso-.-gram is a kind of (-gram, which expresses) 'telegram', as well as for various other bound forms classified earlier as MFCFs: horror.-meister is a kind of (-meister, which expresses) 'expert'.

With endocentricity of suffixations and FCF compounds established conceptually, in a content-based way, this variety is best described as conceptual endocentricity. Suffixations display subordinative conceptual endocentricity, because in the output word the head carries less structural and semantic weight than the modifier, as in legal.-ize, while FCF compounds display coordinative conceptual endocentricity, because in the output word the head and its modifier carry roughly equal structural and semantic weight, as in morpho-.-logy. Again, this property of FCFs is comparable to that of free bases in ordinary compounds.

\section{Summing up}

In an attempt at shedding new light on the phenomenon of headhood of suffixes and FCFs in English word formation, this paper has presented and exemplified their morphological, phonological, syntactic and semantic aspects. In the discussion, a distinction between classical and modern FCFs has been made, as well as between classical, modern and dummy linking vowels. An account has been offered of dual headedness of com- 
posite FCFs, in which a micro-head and a macro-head can be observed to interact. Finally, a content-based approach to suffix/FCF headhood has been proposed, together with the notion of conceptual endocentricity stemming therefrom.

\section{References}

Adams, Valerie 1973. An introduction to Modern English word formation. Longman, London.

Adams, Valerie 2001. Complex words in English. Pearson Education, Harlow.

Back, Gernot 1991. Combining Forms im Englischen. Ms. http://home.arcor.de/gernotback/Magister/Combining_Forms.html.

Bauer, Laurie 1983. English word-formation. Cambridge University Press, Cambridge.

Bauer, Laurie 1990. Be-heading the word. In: Journal of Linguistics 26 :1-31.

Bauer, Laurie 1998. Is there a class of neoclassical compounds, and if so is it productive? In: Linguistics 36 : 403-22.

Cruse, David A. 1986. Lexical semantics. Cambridge University Press, Cambridge.

Dalton-Puffer, Christiane - Ingo Plag 2000. Categorywise, some compound-type morphemes seem to be rather suffix-like: On the status of -ful, -type, and -wise in present day English. In: Folia Linguistica 34:225-44.

Huddleston, Rodney - Geoffrey K. Pullum 2002. The Cambridge grammar of the English language. Cambridge University Press, Cambridge.

Jones, Daniel 2006. Cambridge English pronouncing dictionary (17th edition). Edited by Peter Roach, James Hartman and Jane Setter. Cambridge University Press, Cambridge.

Kastovsky, Dieter 1986. Problems in the morphological analysis of complex lexical items. In: Acta Linguistica Hungarica 36:93-107.

Kemmer, Suzanne 2003. Schemas and lexical blends. In: Hubert Cuyckens - Thomas Berg - René Dirven - Klaus-Uwe Panther (eds): Motivation in language. Studies in honor of Günter Radden, 69-97. John Benjamins, Amsterdam \& Philadelphia.

Lehrer, Adrienne 1998. Scapes, holics, and thons: The semantics of English combining forms. In: American Speech 73:3-28.

Ljung, Magnus 2003. Making words in English. Studentlitteratur, Lund.

Marchand, Hans 1969. The categories and types of present-day English word-formation: A synchronic-diachronic approach (2nd edition). C.H. Beck, München.

McArthur, Tom (ed.) 1992. The Oxford companion to the English language. Oxford University Press, Oxford.

Merriam-Webster, Dictionary 2003. Merriam-Webster's collegiate dictionary (11th ed). CD-ROM. Merriam-Webster, Springfield MA.

Plag, Ingo 2003. Word-formation in English. Cambridge University Press, Cambridge.

Prćić, Tvrtko 1999. The treatment of affixes in the "big four" EFL dictionaries. In: International Journal of Lexicography $12: 263-79$. 
Prćić, Tvrtko 2005. Prefixes vs initial combining forms in English: A lexicographic perspective. In: International Journal of Lexicography 18:313-34.

Quirk, Randolph-Sidney Greenbaum-Geoffrey Leech-Jan Svartvik 1985. A comprehensive grammar of the English language. Longman \& New York, London.

Random, House 1999. Random House Webster's unabridged dictionary (3rd ed.). CDROM. Random House, New York.

Stein, Gabriele 1977. English combining-forms. In: Linguistica $9: 140-7$.

Tournier, Jean 1993. Précis de lexicologie anglaise. (3rd ed.). Nathan, Paris.

Warren, Beatrice 1990. The importance of combining forms. In: Wolfgang U. DresslerHans C. Luschützky - Oskar E. Pfeiffer-John R. Rennison (eds): Contemporary morphology, 111-32. Mouton de Guyter, Berlin \& New York.

Wells, John C. 2000. Longman pronunciation dictionary. Longman/Pearson Education, Harlow.

Williams, Edwin 1981. On the notions 'lexically related' and 'head of a word'. In: Linguistic Inquiry $12: 245-74$. 